\title{
Le glissement de Vajont, ses enseignements \\ et ses retombées pour EDF et les exploitants de barrages
}

\section{G. CASTANIER}

EDF - Chef du service Géologie-Géotechnique 905, avenue du Campde-Menthe 13097 Aix-en-Provence Cedex 02

La catastrophe liée au glissement de Vajont dans le nord de I'Italie (1963) quatre ans après la catastrophe liée à la rupture du barrage de Malpasset dans le sud de la France (1959), a fait prendre conscience des risques liés à la géologie aux concepteurs de barrages et à l'Administration qui a été amenée à créer une réglementation adaptée et à la faire évoluer dans le temps. Sa mise en application, pour les retenues EDF, est illustrée en fin d'article.

Mots-clés : barrages, réservoirs, stabilité des pentes, glissements de terrain, glissements de type banc sur banc, réglementation.

\section{The lesson learnt from the Vajont slide disaster and the consequences for EDF and the dam owners}

The Vajont slide disaster in northern Italy (1963) following the failure of the Malpasset dam four years later in the south of France (1959) made the dam designers conscious of the geological hazards and made the Administration to create a suitable regulation and make it evolve in the time. Its implementation to EDF reservoirs is briefly described at the end of this paper.

Key words: dams, reservoirs, slope stability, rockslides, planar failure along a weak zone, regulation. 


\section{Préambule}

La catastrophe de Vajont s'est produite le 9 octobre 1963, dans les Dolomites italiennes, à $100 \mathrm{~km}$ au nord de Venise. Elle a eu un retentissement mondial et fait l'objet de nombreuses études. Comme toute catastrophe en génie civil, elle a fait progresser les connaissances et a eu une forte influence sur la géologie de l'ingénieur.

Il convient de rappeler qu'avant cette catastrophe, l'étude de la stabilité des pentes au-dessus des retenues de barrages ne faisait pas systématiquement partie des projets et le chantier de Vajont était bien dans ce cas-là.

Les études internationales qui ont tenté d'expliquer le glissement, exceptionnel à de nombreux points de vue, ont soulevé de nombreuses hypothèses et on ne peut pas affirmer, encore aujourd'hui, que toutes les incertitudes ont été levées. La cause n'était pas unique et, comme toujours, ou presque, c'est la conjonction de plusieurs paramètres défavorables qui mène au résultat.

C'est aussi un exemple de l'échec des concepteurs et des géologues à comprendre la nature devant laquelle il convient de rester modeste. Face à la complexité de la configuration géologique du site, on peut encore s'interroger, si un retour dans le temps était possible, sur la probabilité qu'aurait l'erreur de diagnostic de se reproduire encore aujourd'hui.

En fin d'article, sera décrite l'application de la réglementation aux grandes retenues d'EDF en vue de l'établissement des plans particuliers d'intervention (PPI).

\section{2}

\section{Le contexte géologique de Vajont}

Malgré la complexité du site, quelques signes annonciateurs existaient, que les géologues avertis pourraient maintenant relier à ce qui découle du style tectonique d'une région, ou d'une montagne.

Le 22 mars 1959, dans une vallée proche de celle du Vajont, un glissement de terrain produisit une vague dans le réservoir de Pontesei, dont la hauteur atteignit plusieurs mètres au-dessus de la crête du barrage, sans pour autant produire de dégât significatif à l'aval.

On était déjà dans la situation d'un glissement rapide produisant des vagues de hauteur importante.

Il résulta de cet événement, une inspection géologique du réservoir de Vajont confiée à Leopold Müller en juillet 1959.

Celui-ci détecta plusieurs glissements anciens en rive gauche dont un, situé près du barrage (une voûte de $261,6 \mathrm{~m}$ de hauteur), fut jugé potentiellement dangereux ; ce dernier comportait aussi un lambeau visible en rive droite du torrent du Vajont.

Les levées géologiques de E. Semenza en 1959 avaient montré également que le cours actuel du torrent du Vajont est un lit épigénique qui entaille ce glissement ancien qui recouvre, par endroits, des alluvions postérieures à la période glaciaire du Wurm (Fig. 1).

En rive gauche du torrent, les terrains du glissement ancien n'étaient pas désorganisés et le pendage des couches était faible, de l'ordre de $20^{\circ}$ vers l'axe de la vallée.

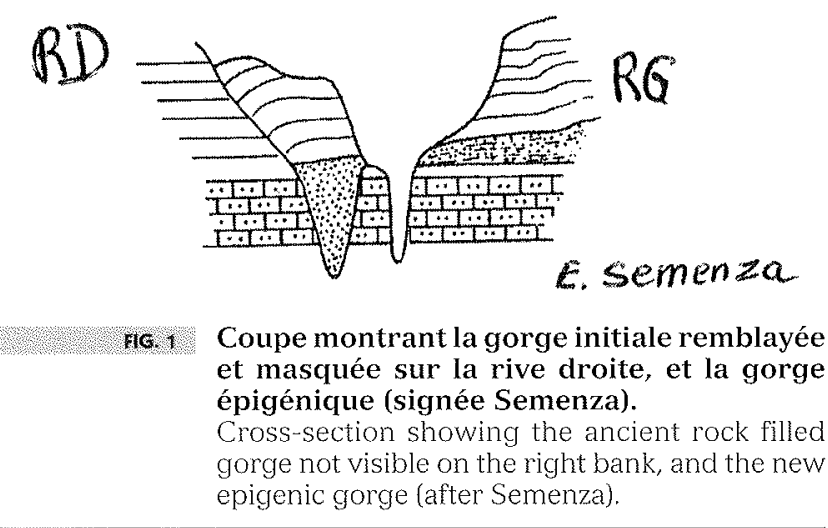

Ces éléments expliquent probablement pourquoi le glissement était passé inaperçu. Le faible pendage des couches est en apparence une configuration géologique plutôt rassurante.

A posteriori, ce glissement fossile est aussi à relier au style tectonique de la région, qui a généré, plus de 10000 ans plus tôt, un énorme glissement qui a remblayé l'ancienne vallée du Vajont.

Une nouvelle fois, on en déduit que l'étude de la géologie régionale est fondamentale pour comprendre la géologie d'un site.

\section{3}

\section{Lhistoire en résumé et l'enclenchement d'un mécanisme inéluctable}

En passant sur les détails, relatés dans de nombreux articles, dont celui de E. Semenza et M. Ghirotti (2000), on va voir qu'une erreur de diagnostic géologique va aboutir à la catastrophe.

En octobre 1960, le remplissage du réservoir finit par révéler les limites d'un glissement ancien, par la matérialisation d'une crevasse longue de $2,5 \mathrm{~km}$ et de $1 \mathrm{~m}$ de large, suite à un mouvement du glissement de $3 \mathrm{~cm}$ par jour (Fig. 2).

On note, sur la figure 2 , le nombre excessivement faible de sondages et de piézomètres, qui laisse entrevoir la mauvaise prise en compte du modèle géologique de glissement.

La figure 3 ci-dessous illustre la méprise qui a abouti à l'erreur de diagnostic.

Le 4 novembre 1960 , alors que le niveau de la retenue était à la cote $650,700000 \mathrm{~m}^{3}$ de matériaux se détachèrent de la partie ouest de l'ancien glissement et créèrent une vague de $2 \mathrm{~m}$ de haut qui s'éleva à $20 \mathrm{~m}$ contre le barrage.

Sans autre information géologique, on peut simplement noter qu'on a affaire, à nouveau, à un type d'instabilité qui génère des vagues comme cela s'était produit dans la retenue de Pontesei.

Mais la réflexion qui s'ensuivit ne prit pas la mesure de ce risque et aboutit à la conclusion que seulement une partition de la retenue était possible. La parade consista alors à réaliser un tunnel de by-pass en rive droite (Fig. 2) après avoir vidangé le réservoir jusqu'à la cote 600 


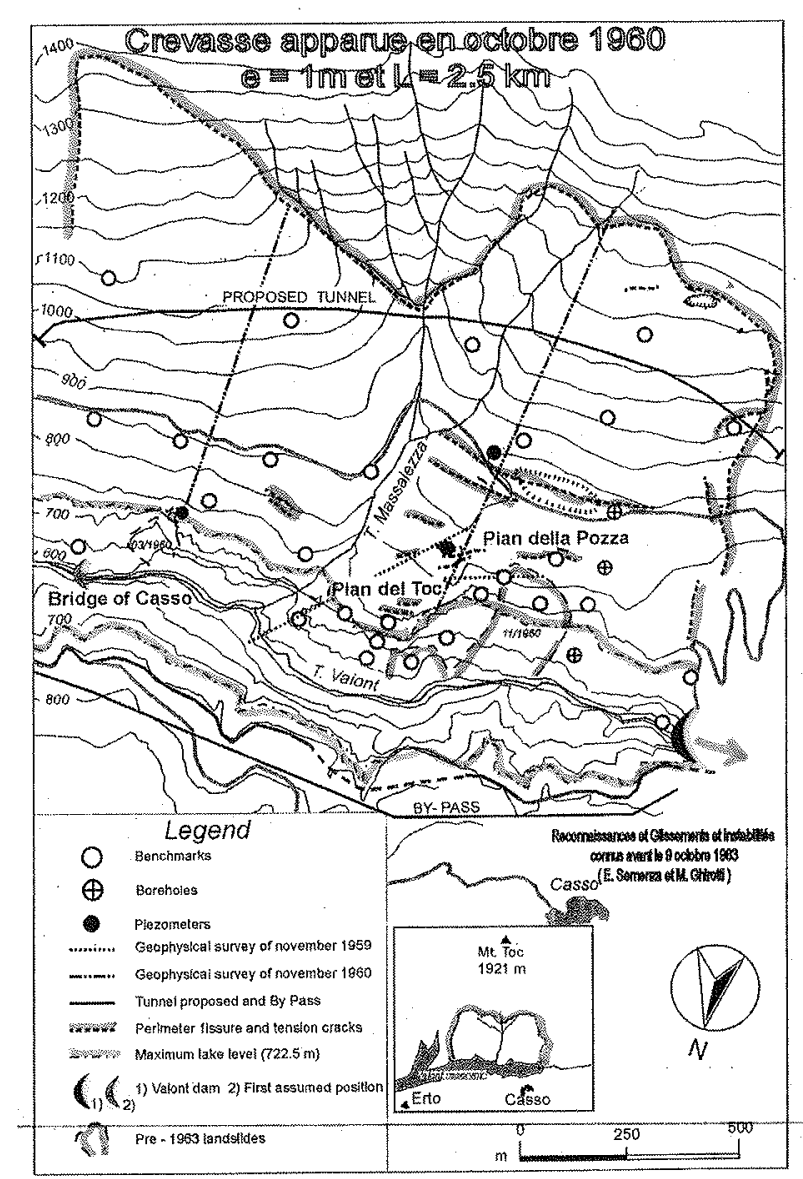

HG. 2 Implantation des fissures et des reconnaissances géologiques : sondages, piézomètres, repères topographiques.

Cracks location and geological surveys : boreholes, piezometers, benchmarks.

Sur la figure 3 , on constate deux pics de vitesse concernant le déplacement du glissement fossile. Les deux pics de vitesse ont été générés par la montée du plan d'eau, d'abord à la cote 650 puis à la cote 700 . Chaque fois la vitesse de déplacement a été annulée par la baisse du plan d'eau; mais le déplacement acquis était conservé.

Après une baisse du plan d'eau, la vitesse de déplacement est beaucoup plus faible lors de la remontée, pour des cotes du plan d'eau égales ou supérieures. Ainsi en décembre 1962, lors de la remontée, quand le lac atteignit la cote 700 , la vitesse de déplacement était de $1,5 \mathrm{~cm} /$ jour alors qu'elle avait atteint $3 \mathrm{~cm} /$ jour à la cote 650 lors du premier remplissage.

CCertains auteurs pensent que le déplacement cumulé depuis la mise en eau de la retenue était compris entre $0,5 \mathrm{~m}$ et $3 \mathrm{~m}$ ).

Ce comportement du glissement fossile a été interprété comme la consolidation d'une masse glissée, saturée pour la première fois par le plan d'eau.

Il n'en était rien, et la troisième reprise de la montée du plan d'eau a été fatale. Le 9 octobre 1963 à 22 h 38, un gigantesque pan de montagne large de $1,6 \mathrm{~km}$ et d'un volume de 240 à $270 \mathrm{hm}^{3}$ glissa dans la retenue qui contenait à ce moment-là 115 hm³ $^{3}$ d'eau.

Le mouvement dura de 20 à 25 secondes. La masse rocheuse parcourut environ $500 \mathrm{~m}$. La vitesse maximale fut estimée à $30 \mathrm{~m} / \mathrm{s}$. Une vague fut poussée sur la rive opposée et atteignit le village de Casso, situé

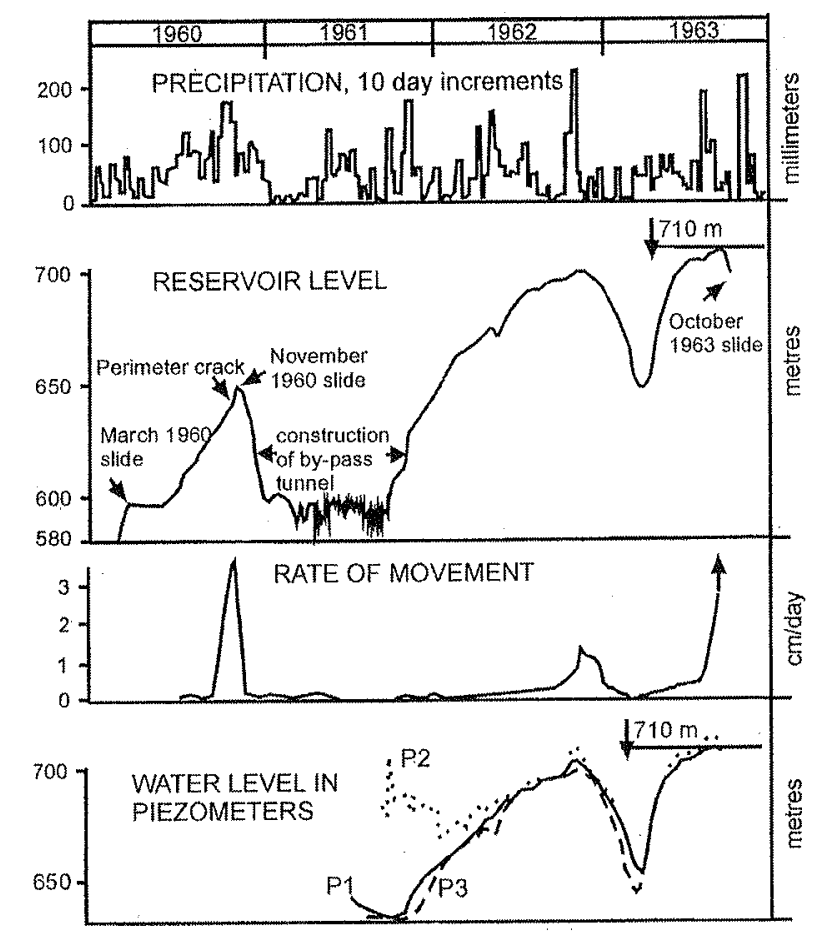

16. 3 Précipitations en mm (par pas de 10 jours), niveau du réservoir en $m$, vitesse $d u$ glissement en $\mathrm{cm} / \mathrm{jour}$, niveaux d'eau dans les piézomètres.

Precipitation, 10 day increment, reservoir level $(\mathrm{m})$, rate of movement $(\mathrm{cm} /$ day) water level in piezometers.

$260 \mathrm{~m}$ au-dessus du lac. $2,5 \mathrm{hm}^{3}$ d'eau furent expulsés du réservoir en donnant une vague estimée à $100 \mathrm{~m}$ de hauteur au-dessus de la crête du barrage. A l'aval, le flot tua 2125 personnes et détruisit 594 habitations.

Les causes avancées par les différents auteurs pour expliquer le déclenchement du glissement et sa vitesse élevée sont nombreuses et varient fortement selon les paramètres privilégiés par les auteurs.

Les paramètres géologiques:

- la structure géologique du versant (Fig. 4) ;

- l'existence d'un ancien glissement ;

- la présence de couches d'argile, de $5 \mathrm{~cm}$ à $15 \mathrm{~cm}$ d'épaisseur, à l'intérieur de la série calcaire, situées au niveau de la surface de rupture et comportant de la montmorillonite (Figs. 5 et 6).

Les facteurs déclenchants :

- le remplissage du réservoir qui déjauge le pied du versant, et ses variations de niveau;

- la sismicité de la région :

- la présence d'un aquifère confiné, captif sous la surface argilisée, peut-être alimenté par une crue karstiques suite à un épisode pluvieux (Fig. 7).

Remarque. La prise en compte du cisaillement interne le long de discontinuités situées à l'intérieur de la masse instable permet d'éviter de recourir à des valeurs trop faibles de l'angle de frottement des couches argileuses (par exemple, $\varphi=5^{\circ}$ à $12^{\circ}$ ), pour expliquer le glissement (Figs. 8 et 9 ). 

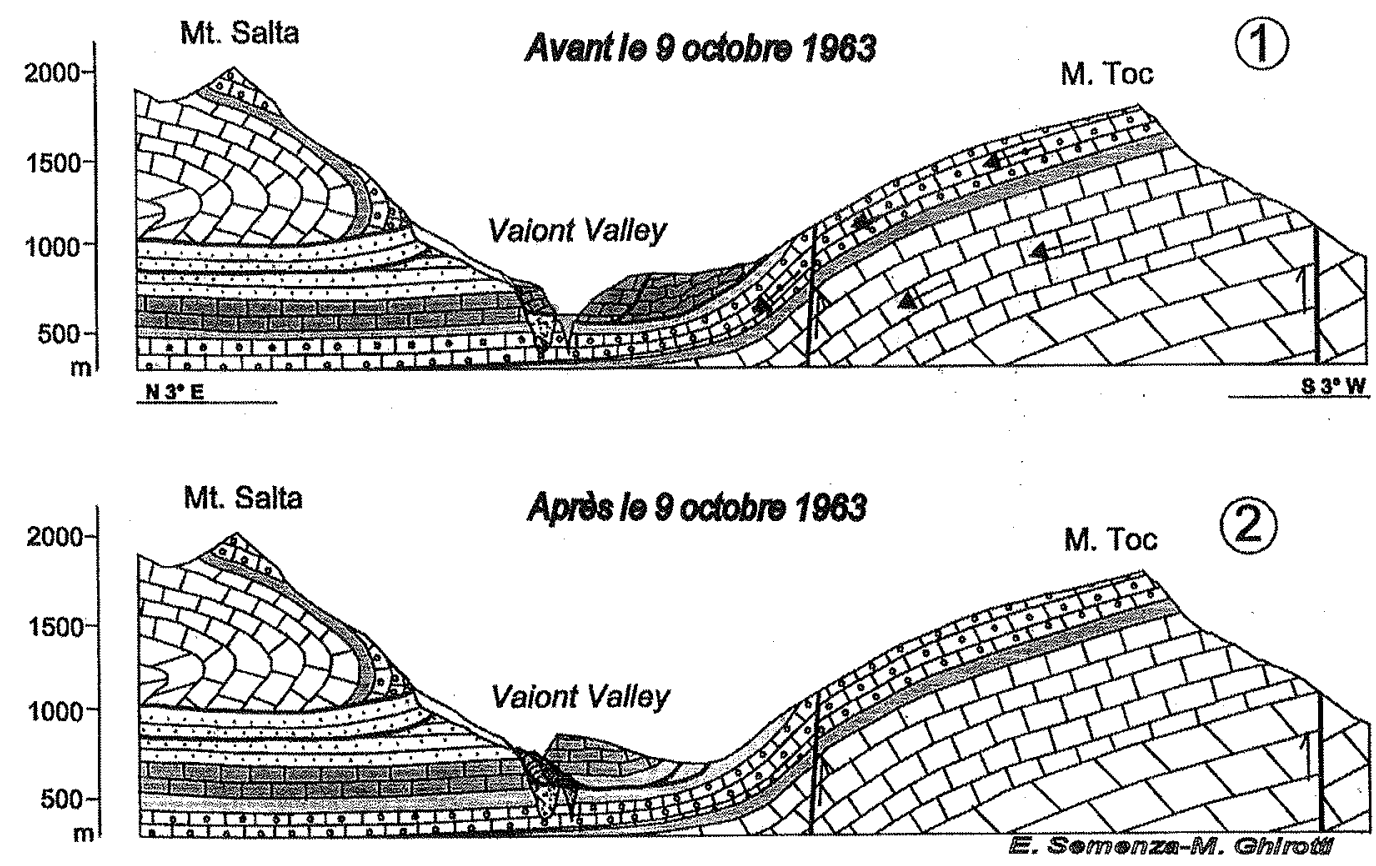

月16. 4 Coupes géologiques avant et après le glissement du 9 octobre 1963. Geological cross-sections before and after October 9th 1963 failure.

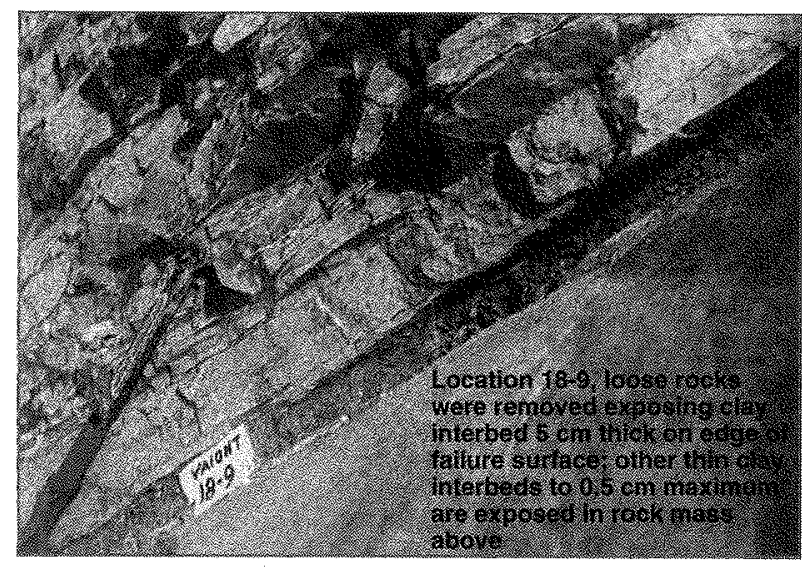

Fa. s. Photo d'une intercalation argileuse de $5 \mathrm{~cm}$ le long de la surface de rupture, d'autres plus minces apparaissent au-dessus (Hendron et Patton).

$5 \mathrm{~cm}$ interbedded clay along the failure surface : other thinner ones exist above.

Pour expliquer la vitesse extrêmement élevée du glissement en fin de course, les auteurs invoquent

- la chute importante de la résistance au cisaillement de l'argile quand la vitesse de cisaillement est élevée, $\left(\varphi=5^{\circ}\right.$ si $\left.V=100 \mathrm{~mm} / \mathrm{mn}\right)$;

- la probabilité pour que les déplacements qui ont précédé le glissement $(0,5$ à $3 \mathrm{~m})$ aient fait passer les caractéristiques mécaniques de l'argile, des valeurs de pic aux valeurs résiduelles;

- l'échauffement de l'eau le long de la surface de cisaillement avec l'augmentation de pression interstitielle correspondante ;

- la formation de coussins de vapeur d'eau, tellement la chaleur dégagée était intense, comparable à celle dégagée par une bombe atomique comme l'a évoqué Pierre Habib dans une note à l'Académie des sciences. Le Professeur Jean Goguel a repris cette hypothèse, maintenant largement admise, pour expliquer certains très

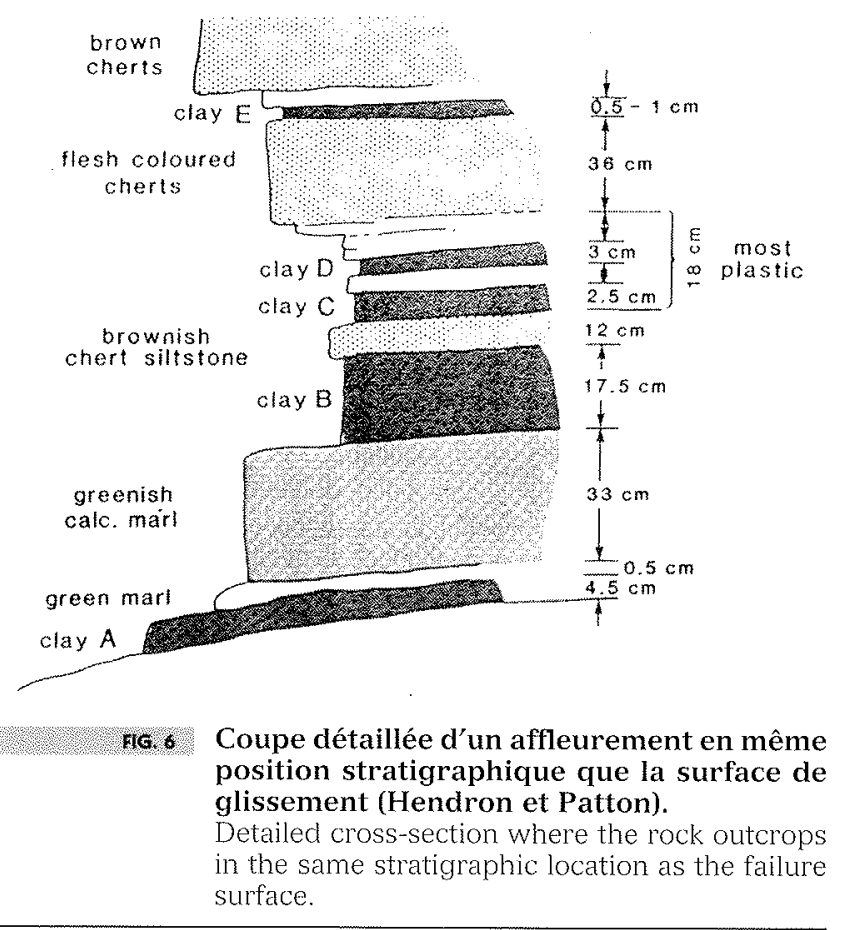

4

\section{Le glissement de type Vajont et ses enseignements pour un maître d'ourrage hydraulique}

Pour un exploitant de grands réservoirs d'eau comme EDF, il convient de recenser tous les paramètres qui conditionnent la dangerosité d'un glissement en termes de vague induite, ou de partition de la retenue et que symbolise le glissement de Vajont. Ce sont : - une masse potentiellement instable, dont le centre de gravité est perché, et où se trouve accumulée une grande énergie potentielle 


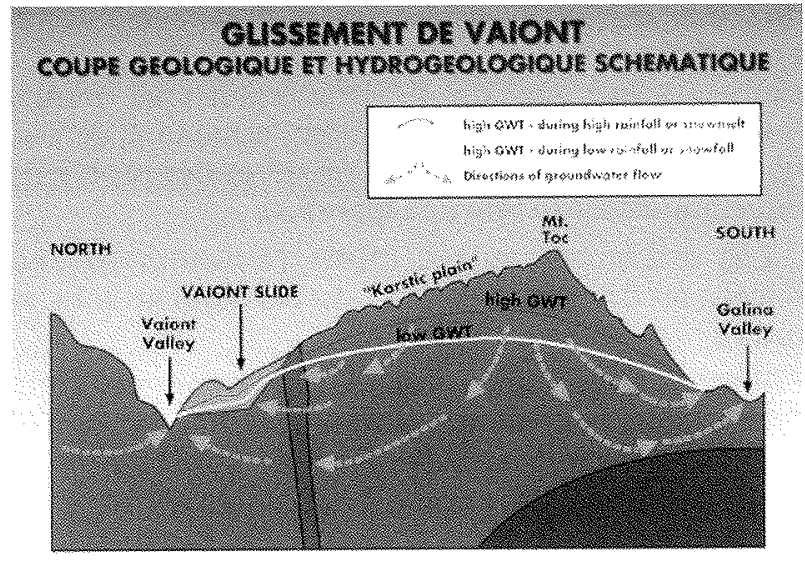

F6. 7 Schéma illustrant l'hypothèse d'une crue karstique générant des sous-pressions sous la surface de rupture.

Diagram showing possible up-lift pressures due to a karstic flood inside the mountain (after Semenza and Dal Cin (1967) in Leonardi and al. (1967)).

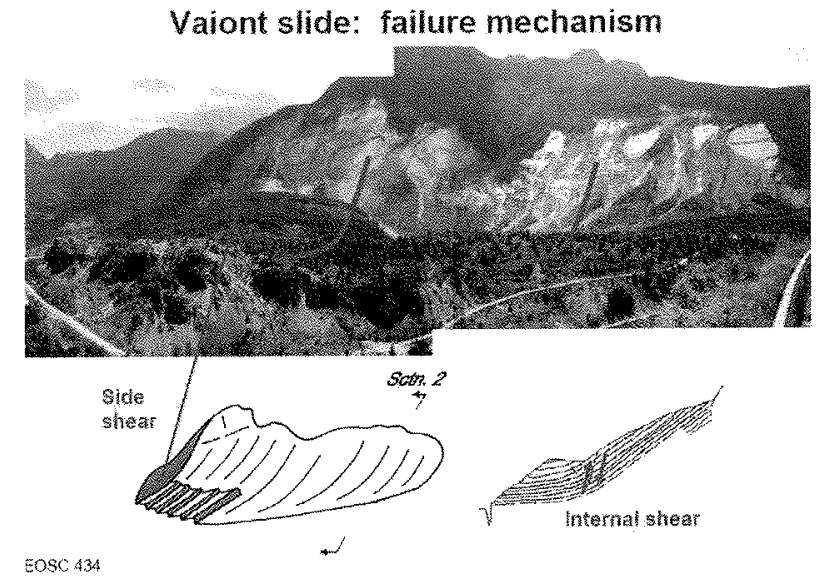

月6. 9 Vue d'ensemble de la cicatrice et du front de la masse glissée.

General view of the scar and the face of the slid mass (after EOSC434).

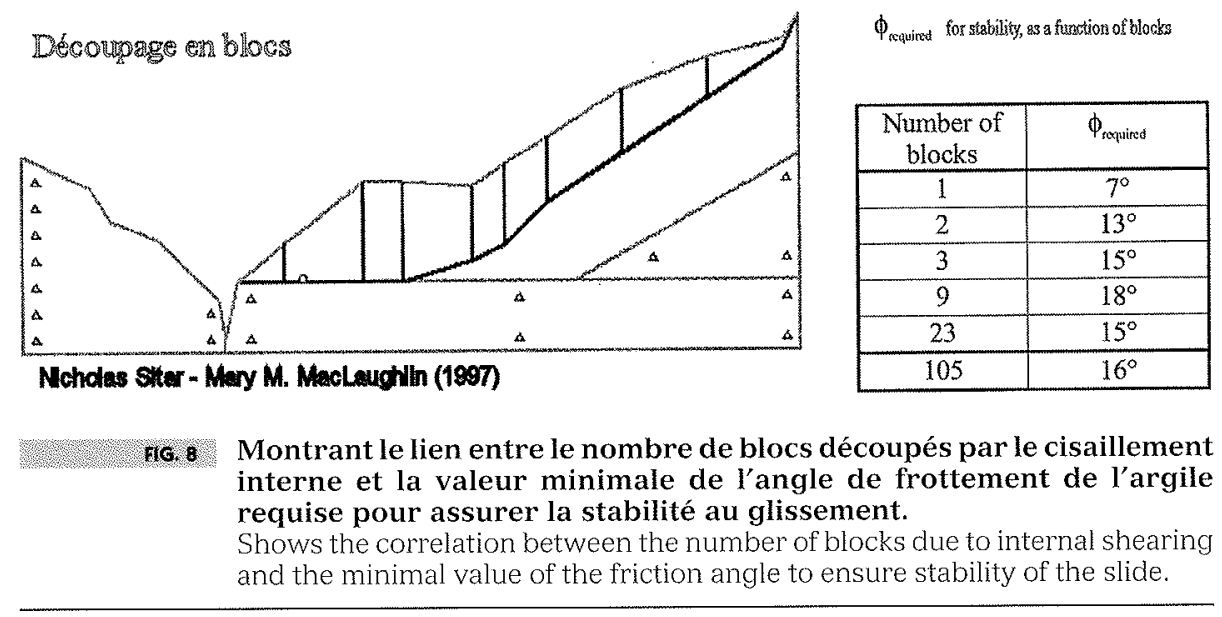

- une configuration géologique de type banc sur banc, c'est-à-dire un massif à forte cohésion à l'intérieur duquel la rupture se concentre sur une zone faible et permettant à une grande partie de l'énergie potentielle de se transformer en énergie cinétique et en chaleur ;

- une vitesse de glissement élevée, résultant du modèle géologique précédent ; à son arrivée dans la retenue, la masse glissée reste monolithique ;

- le volume glissé est gigantesque ;

- le glissement se produit près du barrage.

\section{5}

\section{L'évolution de la réglementation}

\section{(5)}

\section{Création du Comité technique permanent des barrages}

A la suite des catastrophes du barrage de Malpasset en 1959 et du glissement de Vajont en 1963, le gouvernement français a décidé, en 1965, de créer le Comité technique permanent des barrages (CTPB) par le décret du 13 juin 1966. Sa mission a été étendue par le décret du 11 décembre 2007 aux ouvrages hydrauliques importants (d'où le sigle CTPBOH). Il examine tous les barrages « importants pour la sécurité publique » au sens de l'ex-circulaire 70-15, qui ont été assimilés aux barrages de plus de $20 \mathrm{~m}$ de haut au-dessus du terrain naturel (et qui sont maintenant les barrages classés en catégorie A).

Le maître d'ouvrage doit présenter au CTPB un dossier préliminaire en phase " avant-projet sommaire ) (APS) et un dossier définitif en phase " avant-projet détaillé » (APD) qui doit décrire tous les points conditionnant la sécurité de l'ouvrage projeté.

Dans ces dossiers, la partie géologique revêt une grande importance. On y trouve :

- le dossier préliminaire qui décrit en particulier :

- la qualité mécanique des appuis du barrage,

- l'étude géologique des versants ;

- le dossier définitif qui décrit en particulier :

- la géologie générale,

- la sismicité,

- les reconnaissances réalisées,

- la stabilité des fondations,

- l'étanchéité du réservoir,

- la stabilité des rives et des versants. 
Tous les grands barrages EDF datant de l'épopée de l'hydraulique et construits après 1966 sont donc passés deux fois devant le CTPB.

Après le développement du parc nucléaire, parmi les barrages dont le réservoir est rempli par pompage, le dossier du barrage de Vieux-Pré, dans les Vosges, est même passé trois fois devant le CTPB, suite à la nécessité d'adapter l'étanchéité de la fondation aux singularités géologiques apparues à l'ouverture des fouilles.

\section{0}

\section{Les études de danger pour les plans particuliers d'intervention " barrages "}

Initialement, la loi du 22/07/1987, relative à l'organisation des secours en cas de catastrophe, prévoyait l'établissement de plans d'urgence par les pouvoirs publics. Les décrets d'application des 6/05/1988, 15/09/1992 et l'arrêté du 22/02/2002 définissaient les dispositions applicables aux barrages en vue de l'établissement des plans particuliers d'intervention (PPI).

Actuellement, la loi de 1987 a été remplacée par la loi du 13/08/2004 et les décrets du 13/09/2005 et du $12 / 10 / 05$.

Elle concerne les barrages ayant à la fois plus de $20 \mathrm{~m}$ au-dessus du terrain naturel et un réservoir de plus de $15 \mathrm{hm}^{3}$.

Pour permettre aux préfets d'établir ces plans particuliers d'intervention, les maîtres d'ouvrage doivent fournir :

- une analyse des risques que les crues, les séismes ou les effondrements de terrain dans la retenue peuvent engendrer pour les barrages;

- un projet d'installation des dispositifs techniques de détection et de surveillance, et d'alerte aux autorités et aux populations;

- une estimation des conséquences de la rupture de l'ouvrage (onde de submersion).

Une centaine de barrages étaient alors concernés en France parmi lesquels 67 étaient exploités par EDF.

Les études de danger engagées dans le cadre des dispositions nouvelles sur les plans particuliers d'intervention ont été l'occasion pour EDF de reprendre les études pour tous les barrages concernés en adoptant une méthodologie garantissant l'homogénéité des résultats. Les dossiers ont été présentés au CTPB

Les choix effectués et les principaux résultats obtenus sont succinctement décrits ci-dessous pour les glissements de terrain.

\section{6}

\section{L'aléa glissement de terrain}

On distingue trois effets possibles pour le barrage d'un effondrement de terrain dans une retenue :

- la création d'une vague pouvant submerger le barrage ;

- l'impact direct sur le barrage pouvant endommager des organes vitaux de l'ouvrage (vannes, bâtiment de commande...);
- la création d'un ( barrage ) naturel dans la retenue, par partition (le mouvement de terrain vient boucher la retenue jusqu'à une cote supérieure à la cote normale d'exploitation) ou par obstruction (la hauteur de bouchon est inférieure à la cote normale, et des problèmes peuvent apparaitre lors de vidanges).

Les éventuels autres effets (notamment ceux concernant des tiers à l'amont du barrage) ne sont pas étudiés.

Électricité de France possède une expérience consiclérable dans la gestion des mouvements de versants du fait de l'importance de son parc hydroélectrique comportant des sites parfois sensibles. Cette expérience couvre :

- la gestion de mouvements de terrains déclarés;

- la gestion préventive de glissements potentiels susceptibles de mettre à mal la sûreté des ouvrages.

Au cours des années 90, cette expérience a été enrichie par les connaissances acquises par EDF sur des aménagements hydroélectriques en Argentine, dans les Andes. Les retenues y sont implantées dans des formations volcaniques et volcano-sédimentaires hétérogènes, affectées par de nombreux glissements de terrain.

La démarche débute toujours par une étude géologicque précise dont les objectifs sont multiples :

- déterminer les caractéristiques géométriques de la zone instable : limites en surface et volume;

- déterminer la nature géologique des formations instables, de façon à en apprécier la cohésion ;

- rechercher l'existence ou non de surfaces de glissement;

- comprendre l'hydrogéologie du massif;

- apprécier le caractère monolithique ou, au contraire, très fragmentaire de la zone instable;

- typer la zone instable de façon à la rattacher à un type de glissement connu ;

- évaluer les éléments topographiques pour apprécier l'énergie potentielle accumulée au niveau de la zone instable et pour apprécier l'énergie cinétique mobilisable;

- évaluer l'ordre de grandeur de la vitesse de glissement lors de son arrivée dans le lac;

- estimer l'ordre de grandeur de la hauteur de la vague créée, lors de l'arrivée dans le lac. Celui-ci dépend de la vitesse et du caractère monolithique de la zone instable ;

- apprécier le caractère en cours, ou plus ou moins imminent, de l'instabilité du mouvement de terrain.

Le diagnostic géologique peut conclure à l'innocuité de la zone instable, ou à sa dangerosité. Il peut aussi conduire à une demande d'auscultation, des reconnaissances, des calculs de stabilité plus ou moins sophistiqués, des travaux : création de butées de pied ou travaux de drainage, en surface ou par des drains forés à partir de galeries.

L'une des principales difficultés de l'expertise géologique consiste à apprécier le caractère plus ou moins imminent de la rupture; en d'autres termes, si une certaine évolution des paramètres géologiques est nécessaire pour rompre un équilibre métastable. 


\section{Application de la démarche plans particuliers d'intervention aux retenues EDF}

II s'agissait de faire le lien entre les types d'instabilité recensés et la proximité de la retenue.

La démarche suivie par EDF pour ce type d'étude a comporté :

- une phase de collecte et d'analyse des données existantes, concernant les éventuels mouvements de terrain connus ou potentiels, sur le pourtour de la retenue ou à sa proximité. Ces données sont disponibles soit dans des documents internes à EDF, soit auprès d'organismes extérieurs (RTM, DDE, CETE, SNCF, etc.) ;

- une phase de terrain consistant à rassembler le maximum d'observations d'ordre géologique, géomorphologique et hydrogéologique, à un instant donné. Certains indices de surface et morphologiques permettent d'identifier des zones susceptibles d'évolution. Les moyens nécessaires à cette phase de terrain peuvent être assez lourds : bateau, hélicoptère, intervention acrobatique en falaise... Cette phase de collecte des données de terrain a été guidée et complétée par l'analyse de photographies aériennes;

- une analyse de l'aléa ( mouvement de terrain » en fonction de la géologie structurale en grand des versants. En effet, une structuration défavorable des formations géologiques dans un versant (plongement des couches dans le sens de la pente, vers la retenue) peut être propice au déclenchement d'un mouvement de terrain en cas de circonstances particulièrement pénalisantes (épisode pluvieux exceptionnel, vidange rapide), tandis qu'une structuration favorable (plongement vers l'intérieur du massif, s'il n'y a pas fauchage) permet d'écarter virtuellement toute potentialité de mouvement de moyenne à grande ampleur.

Chaque zone potentielle de mouvement a fait l'objet d'un repérage sur un fond topographique au 1/10 000 (Fig. 10) recensant également tous les indices et observations relatifs à la zone, et d'une fiche descriptive et analytique autoportante. Sur cette fiche figure l'estimation de tous les paramètres évoqués ci-clessus, en particulier les éléments géométriques de la zone instable, la potentialité d'occurrence du glissement, y compris clans la retenue, la vitesse probable d'arrivée dans la retenue, la nature du risque (partition ou vague), etc.

Le rôle de la retenue sur le comportement d'une zone potentiellement instable est signalé, le cas échéant. L'analyse de ce rôle peut parfois conduire à des consignes particulières d'exploitation (limitation de la cote du marnage, etc.).

Les différentes zones de mouvements potentiels ont été classées selon la potentialité d'occurrence d'un glissement dans la retenue suivant une échelle de trois couleurs, définie comme suit

- potentialité forte (en gris foncé). La structure et l'état actuel des matériaux sont tels que la modification d'un paramètre (pression interstitielle, cohésion, etc.) peut suffire à la mise en mouvement des masses instables et provoquer leur arrivée dans la retenue ;

- potentialité moyenne (en gris clair). Une certaine évolution de la zone instable est nécessaire, mais possible, pour se retrouver dans le cas précédent;

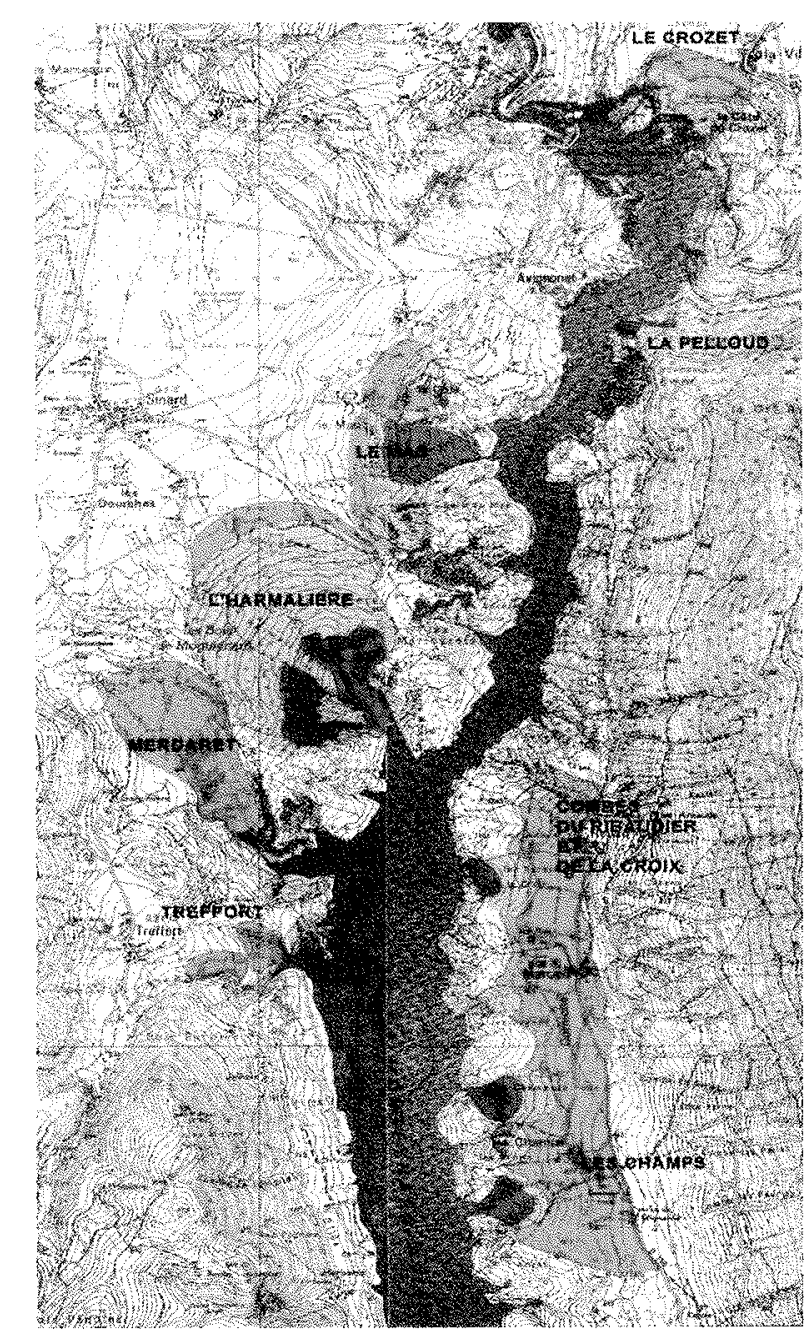

16. 10 Retenue EDF de Monteynard (Isère) cartographie des zones instables.

Monteynard EDF reservoir (Isère) unstable area mapping.

- potentialité faible (en gris moyen). Ces zones sont reconnues sujettes à des mouvements, ou potentiellement instables, mais :

- soit leur configuration réduit considérablement le risque d'arrivée jusqu'à la retenue (distances importantes),

- soit elle induit un étalement sur le plan spatial (disposition géométrique des matériaux) et/ou temporel (évolution très lente),

- soit elle conduit à l'arrivée dans la retenue de volumes très limités (de l'ordre de quelques centaines de $\mathrm{m}^{3}$ )

\section{8}

\section{Les résultats}

Au total, 255 mouvements de terrain déclarés ou potentiels ont été recensés sur les versants des 67 retenues étudiées. $58 \%$ de ces mouvements ont été classés en potentialité d'occurrence dans la retenue faible et $23 \%$ en potentialité d'occurrence forte.

Parmi ces 255 mouvements déclarés ou potentiels, 65 sont suivis : 31 sont surveillés par l'exploitant (surveillance visuelle, prise régulière de photos...) et 34 sont auscultés. La répartition de ces 34 zones auscultées montre que : 
- 19 zones (soit $56 \%$ des cas) sont concernées par l'aléa création d'une vague ;

- 9 zones (soit $27 \%$ des cas) sont concernées par l'aléa partition/obstruction;

- 4 zones soit $12 \%$ sont concernées par l'aléa impact direct sur l'ouvrage ;

- 2 zones sont concernées par des aléas qui n'entrent pas dans le cadre des PPI (stabilité de pylônes, affaissements routiers).

La figure 11 illustre la répartition de ces 255 mouvements de terrain en fonction du type de mouvement mis en jeu et de la potentialité d'occurrence associée, pour les 67 retenues EDF.

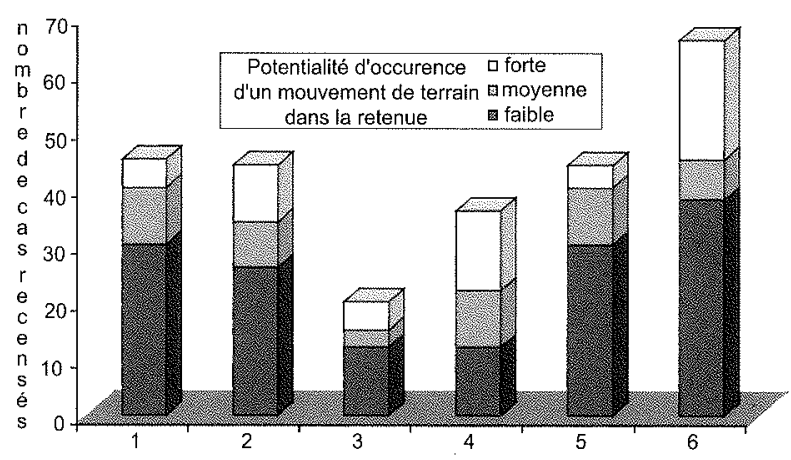

fl6. 1. Répartition des 255 mouvements de terrain déclarés ou potentiels recensés sur les 67 retenues EDF. 1) chutes de blocs ; 2) éboulement par basculement ou cisaillement en pied ; 3) éboulement et glissement banc sur banc; 4) glissements rotationnels ; 5) glissement de matériaux initialement cohérents ayant perdu leur cohésion suite à des phénomènes de versant ; 6) écoulements (fluage, solifluxion ravinement et coulées boueuses).

Distribution of the 255 known or potential slope movements affecting the 67 biggest reservoirs. 1) rockfalls; 2) tilting or rough translationnal slides; 3) planar failure along a weak zone slides ; 4) circular slides ; 5) stides in materials which lost their cohesion in side mountain events weathering, down-bending ; 6) flows (creepings, viscous and muddy flows, gully erosion)

\section{1}

\section{Quelques exemples}

Les chutes de blocs caractérisent des éboulements de masses rocheuses éparses et/ou étalées dans le temps. Le risque associé est un impact direct sur l'ouvrage, ce qui représente une faible proportion des chutes de blocs recensées. L'exemple le plus significatif est celui de l'appui rive gauche du barrage de Pla. de Soulcem (Fig. 12), dans les Pyrénées, qui a nécessité des dispositions particulières de protection du barrage lors de la conception, en cas de perforation du masque et d'alarme par géophones disposés sous le parement du barrage. Les informations, en cas de chute de blocs, sont télétransmises à l'usine hydroélectrique.

Le risque de création de vague pouvan submerger le barrage est associé aux mouvements pouvant mettre en jeu, à de fortes vitesses, des volumes très importants et monolithiques.
Le risque potentiel concernait essentiellement les glissements de type banc sur banc (cas de la Pelloud, retenue de Monteynard dans les Alpes) et les éboulements par basculement/cisaillement de pied de falaise (cas du Chastel, retenue de Puylaurent dans le Massif Central), pour lesquels la cinétique de glissement est brutale et imprévisible. Dans une moindre mesure, il concerne aussi certains glissements rotationnels (cas du Billan, retenue de Grand-Maison, dans les Alpes) associés à des phénomènes de versant ayant conduit à une perte de la cohésion en grand du massif (cf. C. Thomaïdis et al., 2001).

A titre d'exemple, concernant la configuration de glissement de type banc sur banc, on citera le promontoire de La Pelloud (Fig. 13) situé à $1500 \mathrm{~m}$ à l'amont du barrage de Monteynard sur le Drac. Les plans de ( stratification », pentes de $45^{\circ}$ à $50^{\circ}$ vers la retenue, laissaient craindre une possibilité de glissement banc sur banc d'un volume monolithique de plusieurs centaines de milliers de $\mathrm{m}^{3}$, non buté en pied.

Le système d'auscultation anciennement mis en place, qui consistait en un réseau d'observation angulaire avec des mesures de périodicité annuelle, et un réseau de nivellement avec des mesures de périodicité quinquennale, se révéla inadapté à l'aléa.

Une reconnaissance en falaise (Fig. 14) de la nature et de l'état de la stratification fut décidée. Elle révéla que les hypothétiques plans de glissement étaient en fait des joints fermés, recristallisés par de la calcite, recoupés par la schistosité verticale et disparaissant localement entre les plans de schistosité et de fractures. Ces joints, très ténus, sont pratiquement invisibles du haut de la falaise, et ne demeuraient décelables que depuis la rive opposée, car ils sont soulignés par un léger sous-cavage de gélifraction.

Le modèle de glissement banc sur banc put par conséquent être écarté, car les plans de glissement, ondulés et très rugueux en grand, présentent un angle de frottement et une cohésion suffisants. L'intervention en falaise, délicate à mettre en œuvre, se révéla donc très efficace puisqu'elle permit d'éviter des investigations lourdes et coûteuses.

A l'inverse, le glissement du Billan (Fig. 15), apparu en rive droite de la retenue du barrage de Grand Maison dans l'Isère, lors de la mise en eau de la retenue, a dû être stabilisé par un voile de drainage réalisé à partir d'une galerie. Il s'agit d'un glissement de type rotationnel affectant un versant rocheux dont la cohésion a été amoindrie par d'importants phénomènes de fauchage de versant.

Le risque d'obstruction/partition existe pour tous les types de glissements, mais il concerne surtout les glissements rotationnels dans les matériaux meubles et les écoulements (coulées de boues, solifluxion...) situés en bordure de retenues étroites et peu profondes.

En général, les vitesses et les volumes élémentaires demeurent trop faibles pour présenter un risque réel pour l'ouvrage. L'impact des mouvements de versant se traduit alors par un alluvionnement et une perte de la capacité utile du réservoir. C'est par exemple le cas des glissements en loupes imbriquées, dans des argiles glacio-lacustres du tiers amont de la retenue de Vouglans sur l'Ain (Fig. 16), pour laquelle on a vu des sapins transportés au milieu du lac, sur une loupe de glissement, sans générer la moindre vague. 


\section{RETENUE de SOULCEM Appui rive gauche du barrage}
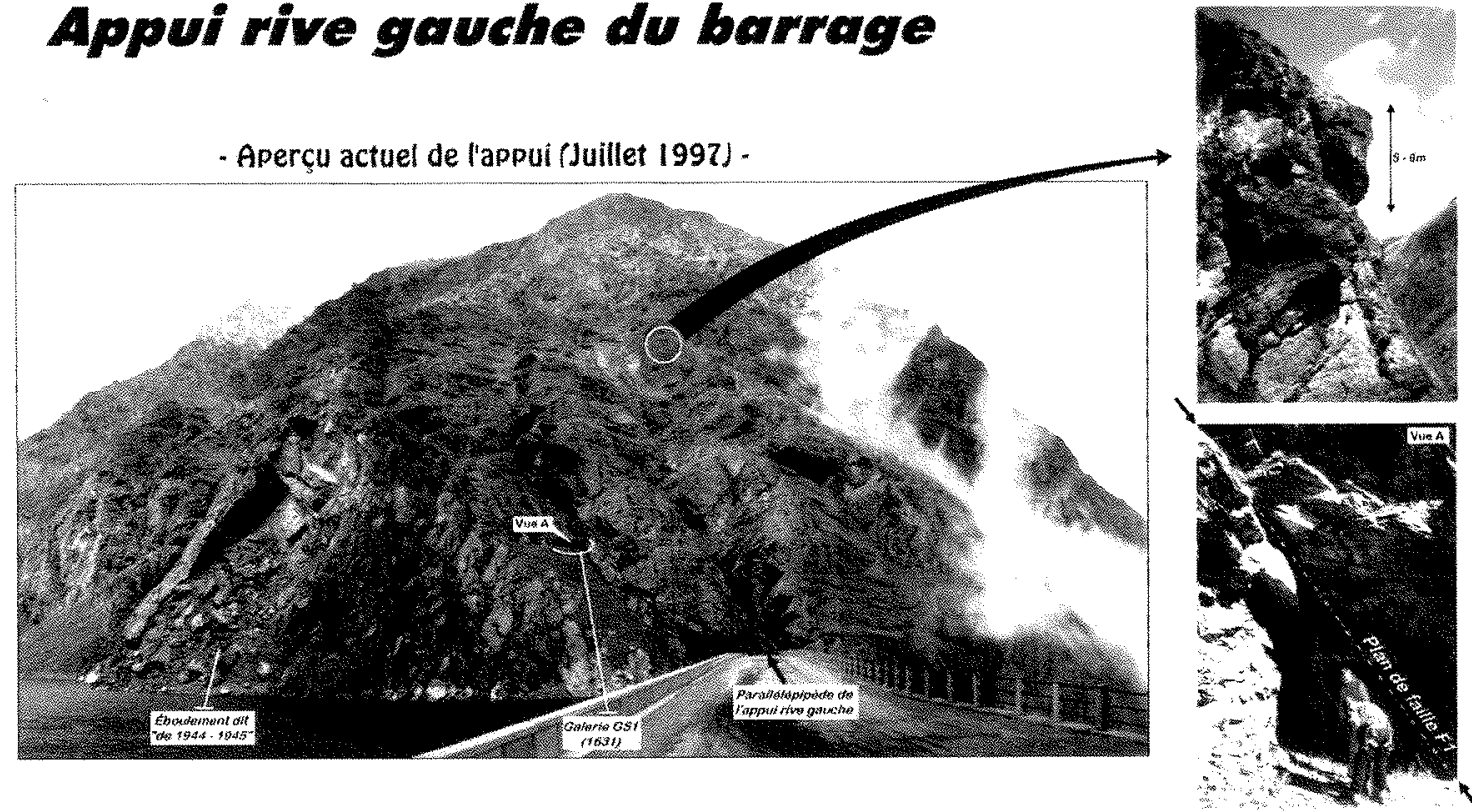

116. 12. Barrage de Pla de Soulcem (EDF) ; blocs perchés au-dessus du masque amont en béton du barrage.

Pla-de-Soulcem EDF dam; blocks above the concrete face dam.

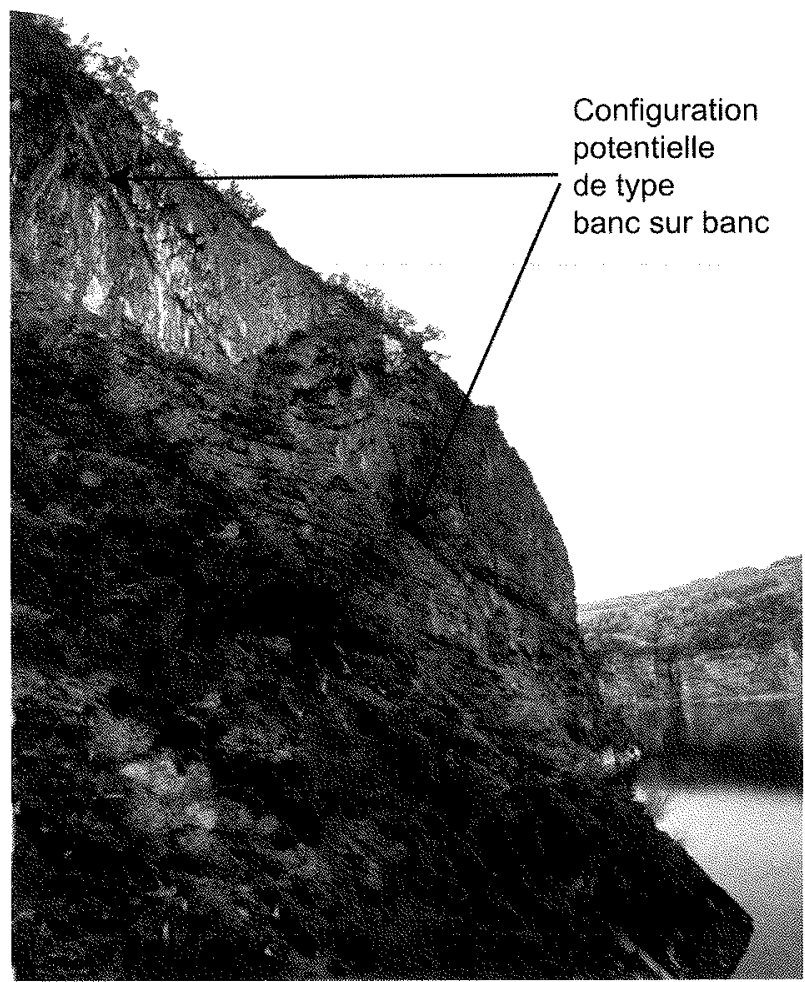

16. 13. Retenue EDF de Monteynard, zone de La Pelloud. Configuration initialement envisagée de type banc sur banc.

EDF Monteynard reservoir, La Pelloud area. Planar failure along a clayey zone initially supposed.

\section{9}

\section{Gestion du risque mouvements de terrain à EDF}

L'approche d'EDF, en cas de crise, repose sur les grands principes suivants :

- rapidité de réaction conduisant, à partir d'un premier diagnostic géologique, à la mise en place d'une auscultation sommaire, adaptée au modèle géologique de glissement,

- diagnostic géologique approfondi conduisant, si nécessaire, à des reconnaissances et des modélisations ;

- le cas échéant, adaptation des consignes d'exploitation de la retenue aux résultats de l'étude;

- mise en place d'un système d'alerte sur la zone instable ou dans la zone menacée, avec définition de seuils d'alerte ;

- en cas de nécessité, définition de travaux de confortement ou de drainage.

Les actions proposées par EDF dans la gestion des mouvements de versants sur les grands ouvrages hydroélectriques sont systématiquement soumises à l'approbation du Comité Technique Permanent des grands Barrages (CTPB maintenant CTPBOH).

L'exploitant du barrage est le maillon essentiel du dispositif de gestion du risque. Il recueille les données du dispositif d'auscultation, assure la surveillance visuelle des versants de la retenue qu'il exploite et déclenche, le cas échéant, une procédure d'alerte. Sa sensibilité aux phénomènes de mouvements de versant est donc fondamentale. Il doit être en mesure d'analyser sommairement les données qu'il acquiert et doit être attentif aux indices d'activation (ou de réactivation) d'un glissement de terrain. Des stages de sensibilisation, animés par des géologues, sont régulièrement mis en place dans cette optique. 


\section{RECONNAISSANCE VISUELLE DU VERSANT}

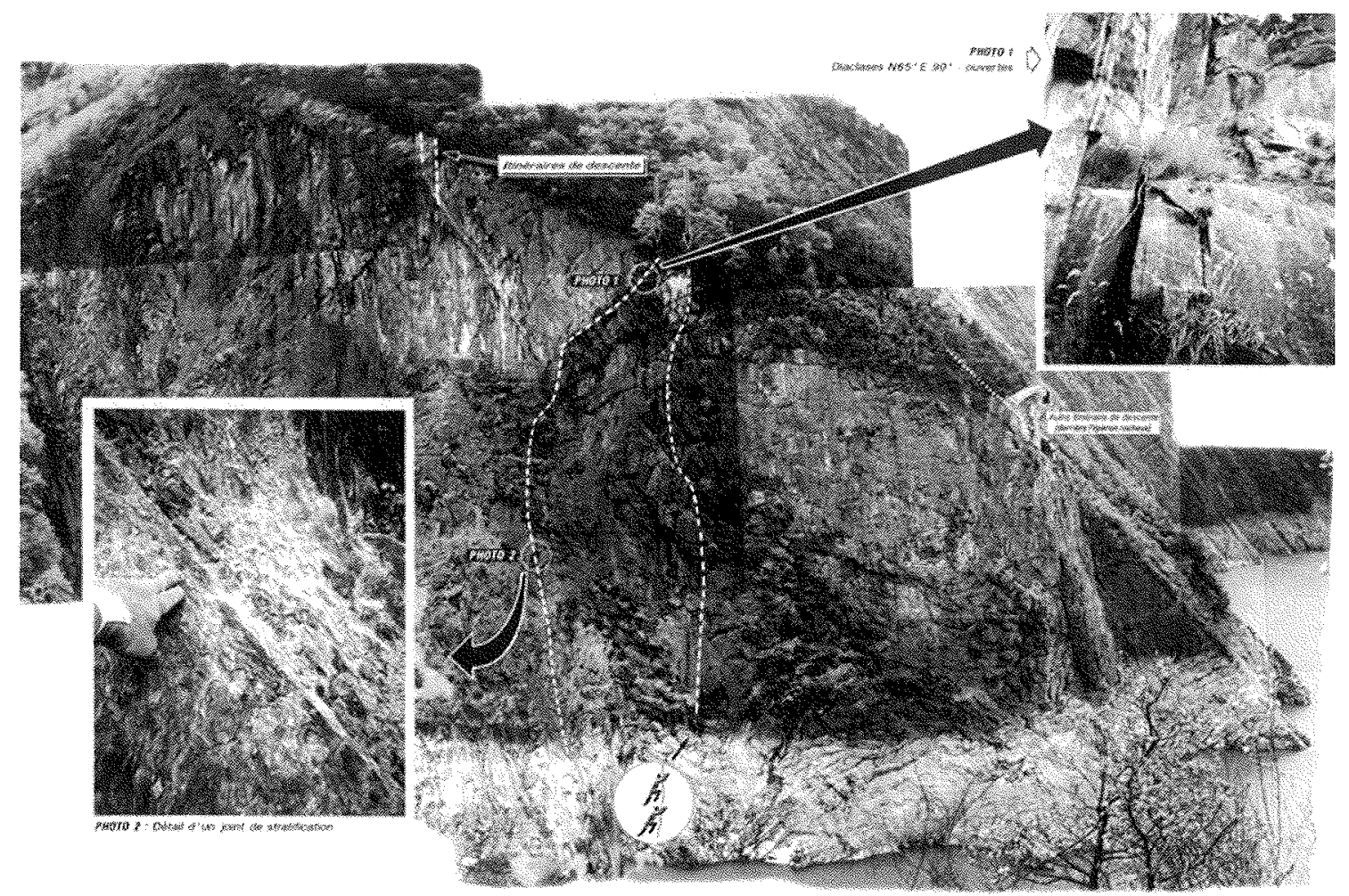

136. 14 Retenue EDF de Monteynard : reconnaissance géologique en falaise du promontoire de La Pelloud.

EDF Monteynard reservoir : geological survey of the La Pelloud cliffs.

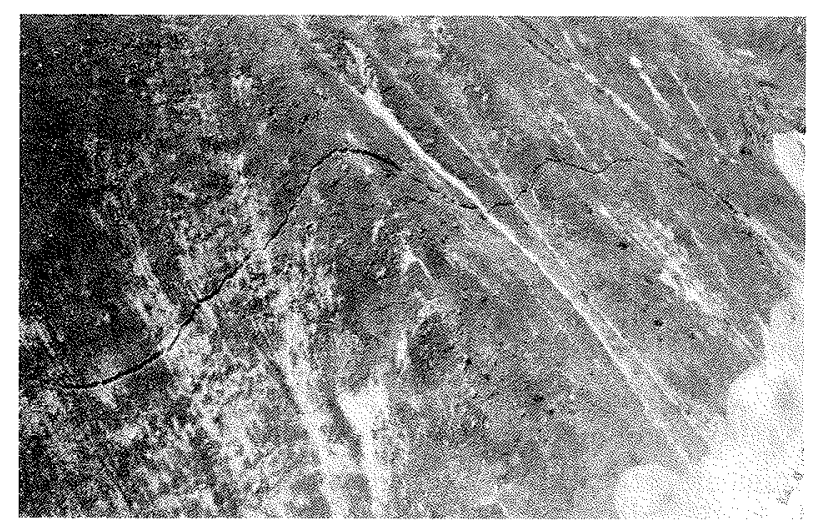

FiG. 15 Retenue EDF de Grand-Maison ; zone instable du Billan, en rive droite, délimitée par la crevasse.

EDF Grand-Maison reservoir ; unstable area on right bank, delimited by the crack.

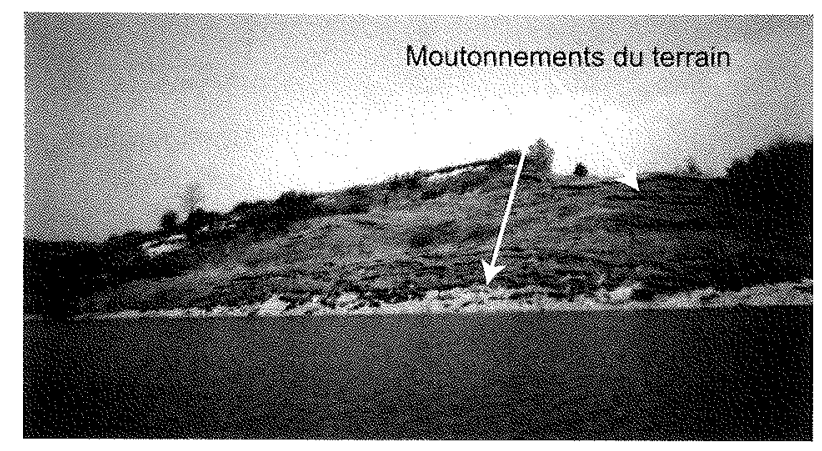

10. 16 . Retenue EDF de Vouglans ; zone instable de la Vourpille.

EDF Vouglans reservoir; La Vourpille unstable area.

\section{Avertissements}

1) II ne doit pas être perdu de vue qu'en géologie, une analyse de stabilité des pentes, fût-elle de type Plan particulier d'intervention, représente l'examen d'un état à un instant donné. Avec le temps, les paramètres géologiques peuvent évoluer soit imperceptiblement, par vieillissement, soit brutalcment, par criscs, au cours de phénomènes météorologiques exceptionnels. Toute étude doit donc être réactualisée si des éléments nouveaux apparaissent, ne serait-ce que pour confir- mer des diagnostics antérieurs, établis à partir d'une science qui n'est pas une science exacte.

2) Les caractéristiques de la vague potentielle générée sont fonction de la vitesse d'arrivée dans la retenue, mais aussi de la surface du front de glissement dans la retenue. Sa propagation dépend de la forme de la retenue. Ces calculs sont délicats et entachés de grandes incertitudes. Pour les cas les plus critiques, des modélisations physiques (modèles réduits hydrauliques) du glissement et de la retenue sont nécessaires. 
Dubié J.Y., Duffaut P. - Management of steep rock slopes after studies on reservoir slopes by Électricité de France. Int. Symp. on Lanslides, Cardiff, 2000

Goguel J. - Rôle de l'eau et de la chaleur dans les phénomènes tectonicues. Rev. Géogr. Phys. Géomorphol. Dynamique, 1969.

Habib P. - Sur un mode de glissement des massifs rocheux. Communication a l'Académie des sciences, 1967.

Fendron A.J., Patton F.D. - The Vaiont slide, a geotechnical analysis based on new geological observations of the failure surface. Department of the Army, US Corps of Engineers, Washington, DC, 1985.

Poupart M., Castanier G. - Plan particulier d'intervention « Barrages »). Synthèse des études de danger à EDF. Colloque $\mathrm{CFGB}$ hébergé par le MATE, Paris, 2003.

Semenza E., Guirotti M. - History of the 1963 Vaiont slide : the importance of geological factors. Bull. Eng. Env., 2000.

Sitar N., McLaughlin M.M. - Kinematics and discontinuous deformation analy- sis of landslide movement. Panamerican Symposium on Landslides, Rio de Janeiro, Nov. 10-14, 1997.

Thomaïdis C., Dubié J.Y., Devèze G. Mouvements de versants des retenues hydroélectriques. Retour d'expérience et gestion du risque. Revue française de géotechnique, no 95-96, 2001, p. 165-176. 